\title{
Some recent advances in the chromatographic analysis of lipids
}

\author{
W.W. Christie \\ The Scottish Crop Research Institute, Invergowrie, Dundee, DD2 5DA, Scotland
}

Procedures for structural analysis of fatty acids and high-performance liquid chromatographic (silver ion, adsorption, reversed-phase and chiral) analysis of lipids are in the forefront of developments. Supercritical-fluid chromatography is making a lesser impact. ny selection of the most interesting and valuable advances in the chromatographic analysis of lipids over the last few years must be a subjective one, conditioned by the experience of the author. With this caveat, I suggest that procedures for structural analysis of fatty acids must be high on any list, and those involving gas chromatography-mass spectrometry in particular. Similarly, methodology involving high-performance liquid chromatography (HPLC) has been developing apace, especially in the area of silver ion chromatography, lipid class analysis and molecular species analysis. The increasing use of evaporative light-scattering detection has greatly assisted with the last. With such instruments, the mobile phase coming from the end of the HPLC column is evaporated in a stream of air in a heated chamber; the solute does not evaporate, but is nebulized and carried as minute droplets through a light beam, which is reflected and refracted. The amount of scattered light is measured by a photomultiplier tube and bears a relationship to the concentration of material eluted from the column. In that they will respond to any lipid that does not evaporate before passing through the light beam, such detectors are universal in their applicability. Commercial detectors, based on this principle, now afford great sensitivity. Applications of such detectors for analysis of lipids have been reviewed recently [1].

There has also been a great deal of interest in the application of supercritical-fluid chromatography to the analysis of lipids. However, in my opinion, there is still little that can be done by this technique that is not better accomplished by GC or HPLC. A comprehensive review of this methodology has also appeared [2].

\section{Structural analysis of fatty acids}

The potential of mass spectrometry for identification of fatty acids of natural origin has been evident for many years, but there were few reports of structural analyses on real samples as opposed to model compounds until relatively recently. The problem has been largely solved by the use of specific amide or ester derivatives, which following ionization in the mass spectrometer stabilize the charge on a nitrogen atom remote from the structural features of interest. Radical-induced cleavage predominates in the aliphatic chain and distinctive fragmentations are achieved according to the position of double bonds, branch-points or ring structures. In particular, the use first of pyrrolide, and subsequently of picolinyl ester or 2-alkenyl-4,4-dimethyloxazoline (DMOX) derivatives, of fatty acids permits GC-MS to be employed for the separation and characterisation of these compounds from natural lipids. The technique has been aided by the availability of improved capillary columns. Indeed, such derivatives only began to be used widely when GC columns of fused silica became available, although initially it was only possible to use non-polar stationary phases; picolinyl esters tend to elute at temperatures about $50{ }^{\circ} \mathrm{C}$ higher than for methyl ester derivatives. However, polar, thermally stable phases (prepared via chemical bonding and cross-linking) are being used increasingly.

If mass spectrometry alone is considered, picolinyl (3hydroxymethylpyridine) esters are the most useful derivatives; they permit a wide range of structures to be identified including fatty acids with branch points as well as double and triple bonds. On the other hand, DMOX derivatives have much better gas chromatographic properties, as they elute at lower temperatures (typically only $10^{\circ} \mathrm{C}$ higher than methyl esters), permitting better resolution, and they can be used with a wider range of polar stationary phases. They can be prepared by a simple one-step procedure, both from free fatty acids and from intact lipids. Their mass spectrometric properties differ somewhat from those of picolinyl esters, and they are especially useful for fatty acids with conjugated double bond systems, and for certain (but not all) ring structures. In my opinion, picolinyl esters and DMOX derivatives should be considered as complementary to each other, not as competitors. The use of ancillary techniques such as deuteration or reaction of double bonds with dimethyl disulphide, or simplification of complex mixtures by silver ion chromatography, can greatly facilitate analysis. Applications to fatty acids have been thoroughly reviewed elsewhere $[3,4]$.

To illustrate the fragmentation of an unsaturated fatty acid as its picolinyl ester, the mass spectrum of the 5,11,14,17eicosatetraenoate derivative, a component of some conifer seed oils, is shown in figure 1 . There is a prominent molecular ion $(\mathrm{m} / \mathrm{z}=395)$, and large ions at $\mathrm{m} / \mathrm{z}=92,108,151$ (McLafferty rearrangement ion) and 164 from the picolinyl ester moiety, which should be present in most spectra. Among the more interesting diagnostic features is a regular series of ions $14 \mathrm{amu}$ apart, starting from the molecular ion, for cleavage between each methylene group in the chain. When a double bond is reached, gaps of 26 amu appear. 
In this instance, such gaps between $\mathrm{m} / \mathrm{z}=366$ and 340,326 and 300, and 286 and 260 confirm the positions of the double bonds in positions 17,14 and 11 respectively. The corresponding ions for the double bond in position 5 are less easy to discern, but experience with model compounds confirms that ions at $\mathrm{m} / z=219$ and 232 are characteristic for this structural feature.

The mass spectra of DMOX derivatives appear to be closer to those obtained from pyrrolides (indeed the molecular weight is identical) than from picolinyl esters. With most, there tends to be a distinct molecular ion, with the base peak at $m / z=113$ (McLafferty rearrangement ion) and another prominent peak at $\mathrm{m} / z=126$. There is then a regular series of ions 14 amu apart for cleavage between each methylene group in the chain, interrupted by structural features such as double bonds or branch-points (Zhang et al. 1988a). Often the high molecular weight ions of diagnostic value are less abundant, but nonetheless clear. Again with the spectrum of the derivative of 5,11,14,17-eicosatetraenoate, there is a reasonable molecular ion. A gap of 12 amu between $\mathrm{m} / z=302$ and 314 (carbons 16 and 17) is indicative of a double bond in position 17. Similar features determine the positions of the other double bonds, except for that in position 5. In this instance, recourse to the spectrum of model compounds is necessary and prominent ions at $\mathrm{m} / \mathrm{z}=153,166$ and 180 are characteristic of a double bond in position 5 (author, unpublished).

DMOX and picolinyl ester derivatives have now been applied to the structure determination of a large number of natural and synthetic fatty acids. DMOX derivatives are not suited to branched-chain or cyclopropanoid fatty acids, but can be used in most other circumstances. Difficulties of interpretation can arise when structural features are close together or are near either end of the molecule. In such circumstances, spectra from authentic model compounds can be very useful for identification, or certain ancillary techniques can be used. For example, deuteration is a useful aid to locating the position of a double bond in a fatty acid, but it is noteworthy that by far the best results are obtained with picolinyl ester derivatives [5,6]. Although a variety of reagents have been used for the purpose, the one favoured nowadays is deuterium gas with Wilkinson's catalyst (tris(triphenylphosphine)rhodium(I) chloride), as it does not cause any scrambling of the double bonds.

As an alternative, for the structural analysis of monoenoic acids especially, the simplest derivative involving direct addition to a double bond consists in the preparation of dimethyl disulphide adducts. It is a simple one-pot reaction and requires only that the methyl ester derivative of the unsaturated fatty acid is reacted with dimethyl disulphide and a catalytic amount of iodine [7]. In the mass spectrum, cleavage occurs predominantly between the carbons that originally formed the double bond and interpretation is straight forward. Such adducts have been used in a number of laboratories to identify unknown monoenes, and even some dienoic fatty acids. Although the common methylene-interrupted dienes cannot be analysed easily in this way, they can again give useful results when there are two or more methylene groups between double bonds [8].

Modern Fourier-transform infrared (FTIR) spectroscopy has a number of advantages in relation to older dispersive methods, and in particular a high signal-to-noise ratio that
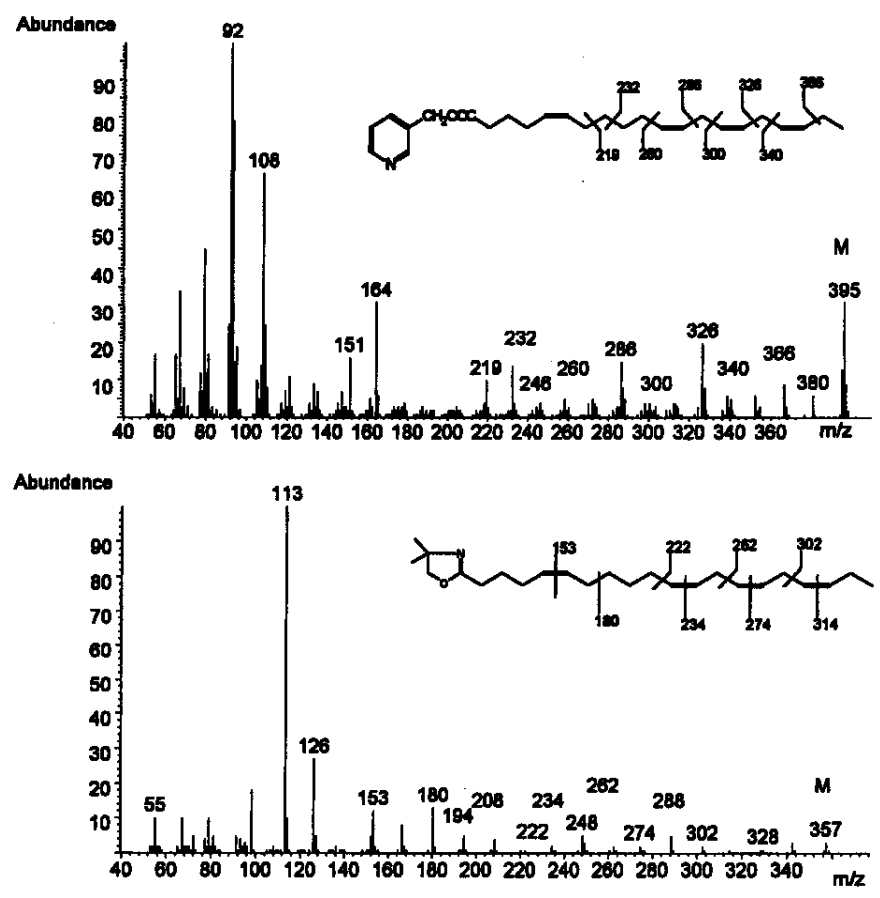

Figure 1. Mass spectra of picolinyl ester (upper) and dimethyloxazoline (lower) derivatives of 5,11,14,17-eicosatetraenoic acids.

permits great sensitivity and good quantification. When linked to gas chromatography, it can be an extremely powerful tool for the determination of fatty acid structure. Mossoba and coworkers in the USA were among the first to establish the value of GC-MI-FTIR, applying it to the analysis of trans-dienes in commercially hydrogenated soybean oil [9]. For example, four trans,trans-dienes were detected and quantified at low levels, including 9-trans,12-trans-octadecadienoic acid. GC with capillary columns had the capacity to effect resolution of the compounds of interest, and the FTIR spectra were invaluable for identification and quantification purposes. In subsequent work, further components were identified by prefractionation of the mixture by silver ion TLC before analysis by GC-MI-FTIR [10].

\section{HPLC separation of lipid classes}

One of the most widely-used applications of HPLC has been for the analysis of phospholipid classes (reviewed in some detail elsewhere [11]). Over the years, the methodology has developed to suit the detection techniques available. Thus, initially the only convenient detectors were UV spectrophotometers operated at low wavelengths, so it was necessary to find combinations of solvents for the mobile phase that were transparent in the appropriate regions of the spectrum. Two basic solvent systems were developed for phospholipid separations that still find almost universal application today, i.e. hexane-propan-2-ol-water and acetonitrile-water (sometimes with added methanol) mixtures. With the latter, phosphatidylethanolamine elutes before phosphatidylcholine and then sphingomyelin, and indeed all the choline-containing 
phospholipids are well resolved. A further special virtue is that the acidic lipids, such as phosphatidylinositol and phosphatidylserine, elute ahead of phosphatidylethanolamine. Mobile phases based on hexane-propan-2-ol-water have also been used in many laboratories. Again, phosphatidylethanolamine elutes before phosphatidylcholine, but the other choline-containing lipids, such as sphingomyelin and phosphatidylcholine, tend to be less well resolved. The acidic phospholipids are separated from each other, but in this instance they emerge between phosphatidylethanolamine and phosphatidylcholine. By adding further solvents to the basic mixture or by using gradients, it has been possible to improve the resolution of the choline-containing lipids especially. This system has proved easier to adapt to simultaneous separation of simple lipids and glycolipids than has that based on acetonitrile. In all the early work, silica gel was the adsorbent, but now chemically bonded phases are finding increasing use.

It is not always recognized that as phospholipids are ionic molecules they require a counter ion in the mobile phase. Sulphuric and phosphoric acids have often been used, but apart from dissolving HPLC equipment they will bring about complete destruction of any plasmalogens present. Ammonia has also been employed although there is some danger that column life could be greatly reduced because silica dissolves at a high $\mathrm{pH}$. A phosphate buffer $(25 \mathrm{mM}, \mathrm{pH} 7.0)$, together with propan-2-ol and hexane, gave excellent results in a method involving UV detection described by Patton et al. [12], that has been adopted in many laboratories. An alternative is to use organic buffers, such as those based on triethylamine-acetic acid.

Modern evaporative light-scattering detectors have fewer limitations. They give good results, even under gradient elution conditions, and are simple and rugged in use; they are not affected by changes in the mobile phase or small variations in ambient temperature or in the flow-rate of the mobile phase. Even when the solvent composition changes rapidly, there is no disturbance of the base-line and little drift during long periods of use. Most solvents, including acetone, propan-2-ol, toluene and chloroform, for example, can be used in the mobile phase, and these can contain up to $20 \%$ water and small amounts of ionic species even. While the minimum limit of detection is to some extent dependent on the nature of the lipid, it is roughly $10 \mathrm{ng}$ in the best commercial instruments.

Although the detector is destructive in that the sample is lost in the current of air, it is possible to insert a stream splitter between the end of the HPLC column and the detector to divert much of the sample for collection. When used in this way, it is an invaluable research tool, as samples can be collected easily for analysis by other methods, such as for fatty acid analysis by GC. A further advantage during the development of new methods is that rapid adjustments to the chromatographic conditions are easy to make, since changing the solvent has almost no effect on the base line. The availability of these detectors has given lipid analysts considerable opportunities to use a wide range of mobile phases, and in complex gradients, to improve the selectivity of particular separations.

An important problem in lipid analysis is the separation of all the main lipid classes, both simple and complex, in tissues in a single chromatographic run. As the structures and polarity of the compounds vary appreciably, this can be a considerable challenge, especially for HPLC. The objective was first accomplished for animal tissues with the aid of a light-scattering detector and a ternary solvent delivery system with a short column $(5 ¥ 100 \mathrm{~mm})$ packed with Spherisorb $^{\mathrm{TM}}$ silica gel (3 $\mathrm{mm}$ particles) [13]. The only constraints in the choice of solvents for the mobile phase were that they should be sufficiently volatile for evaporation in the detector under conditions that did not cause the solute to evaporate, and by a need to avoid inorganic ions, which could not be volatilized. A gradient elution system with eight programmed steps was devised, starting in essence with isooctane or hexane to separate those lipids of low polarity (cholesterol esters and triacylglycerols) and ending with a mobile phase containing water to elute the phospholipids. To mediate the transfer from one extreme to the other, a solvent of medium polarity was required and propan-2-ol-chloroform $(4: 1, \mathrm{v} / \mathrm{v})$ gave the best results. After each analysis, a gradient was generated in the reverse direction to remove most of the water adhering to the silica gel, and to re-establish the activity of the column before the next analysis. Later, it was demonstrated that better resolution of minor acidic components could be obtained by adding small amounts of organic ionic species to the aqueous component of the eluent, e.g. 0.5 to $1 \mathrm{mM}$ serine buffered to $\mathrm{pH} 7.5$ with triethylamine [14].

Although there were abrupt changes in solvent composition at various times, no perturbation of the base-line was apparent, and all the main simple lipid and phospholipid classes of animal tissues were separated from each other in only 20 minutes. After a further 10 minutes of elution to reequilibrate the column, the next sample could be analysed.

The nature of the response of this type of detector has been studied in some detail [15-17], and it is known that it depends both on the nature of the solvent and of the solute. When it is used in quantitative analysis of lipids, it is necessary to determine the optimum conditions for the desired separation first and then carry out a calibration with standards similar in nature to the samples to be analysed. The operating parameters of the detector, mainly the air pressure (and flow-rate) and evaporator temperature, must also be optimised and fixed. For most lipids, the response of the first commercial detector of this type was approximately linear in the range 50 to $200 \mathrm{mg}$, but it tended to fall off rapidly below $10 \mathrm{mg}$; newer models of detector are approximately 100 times more sensitive and the linear range is approximately 0.1 to $10 \mathrm{mg}$. The linearity problem arises because light is scattered much less by small droplets as their diameters near that of the wavelength of light; as detector design has improved, the problem has been reduced but not eliminated. In quantitative analysis, the accuracy and precision of evaporative light-scattering detectors can be improved by adding an internal standard, e.g. phosphatidyldimethylethanolamine has been used in analyses of lipid classes [18].

Methods of this type for the separation of simple lipids and phospholipids or of phospholipids alone, and adapted from the above or similar to it, have now been described from a number of laboratories and confirm that the techno$\log y$ is robust [19-23].

Moreau [23] has published an interesting adaptation of the above method for the analysis of plant lipid classes, where high proportions of distinctive glycolipids are present. 
(A comparison of the use of evaporative light-scattering and transport-flame ionisation was also made; the former was clearly superior). The author [24] has also obtained some good separations of plant lipids by a related method, but with stationary phases containing various organic moieties bonded chemically to silica gel. For example, a separation of the individual simple lipids, glycolipids and phospholipids from a lipid extract of leaves is illustrated in figure 2 . All the main components were clearly separated on a column containing polymerized vinyl alcohol bonded chemically to silica, with a ternary gradient elution scheme.

\section{Silver ion high-performance liquid chromatography}

The first attempts to adapt silver ion chromatography to HPLC met with limited success only (see NikolovaDamyanova [25] for a review). Then, the method was to impregnate an HPLC grade of silica gel with silver nitrate, but silver ions eluted continuously in the mobile phase, contaminating detectors and fractions collected.

The alternative approach adopted by the author was to convert an ion-exchange medium (sulphonic acid groups chemically bonded to silica gel) in an HPLC column to the silver ion form in situ by injection of silver nitrate through the Rheodyne valve, while pumping water through the column, before the aqueous phase was replaced with organic solvents [26]. Although only about $80 \mathrm{mg}$ of silver nitrate was required, this was quite sufficient for practical separations. The simplest elution scheme for analyses of molecular species of triacylglycerols was a gradient of acetone into dichloroethane-dichloromethane [27], which served for fats with a small proportion of linoleic acid, such as sheep adipose tissue or bovine milk fat. This gave resolution of the main components with zero to three double bonds in total in the fatty acyl chains, including separation of fractions with trans- from those with cis-monoenoic residues.

Most triacylglycerol samples are likely to contain appreciable proportions of linoleic acid, and resolution into molecular species was then accomplished by ternary gradient elution, simply by introducing acetonitrile into acetone after the first fractions were recovered. One dienoic acyl moiety was retained more than twice as strongly as a monoene, and one triene $(18: 3(n-3))$ was retained by the same amount as two dienoic residues in a molecule, so there was some overlap of dienoic and trienoic species when a-linolenic acid was present in a sample. Such highly unsaturated triacylglycerols as linseed oil [27] and fish oils [27-29] were fractionated successfully. With the former, trilinolenin was the most abundant single fraction, and a simple progression of fractions with increasing numbers of double bonds were eluted until this species was reached. When the more saturated molecules of fish oils eluted, resolution was excellent and it was perhaps surprising to find appreciable amounts of trisaturated and disaturated-monoene species. Base-line resolution was no longer possible when molecules containing polyunsaturated fatty acids began to elute, because the wide range of positional isomers caused similar components to overlap. Nevertheless, valuable separations of species containing two saturated and/or monoenoic fatty acids and one polyenoic fatty acid especially were achieved.

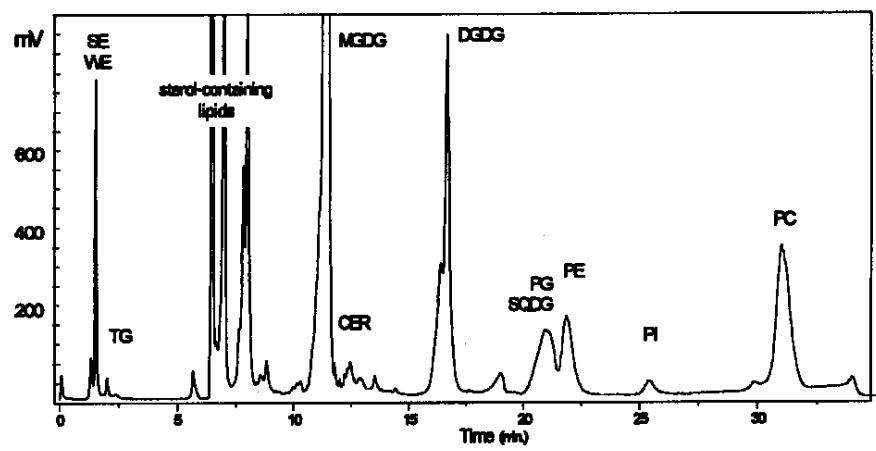

Figure 2. HPLC separation of the complex lipids from leaves of Arabidopsis thaliana, using a ternary-gradient elution scheme with evaporative light-scattering detection on a column of YMC PVA-SilTM (100 $¥ 3.2 \mathrm{~mm}$ ), based on that described by Christie and Urwin [24]. Abbreviations: SE, sterol esters; WE, wax esters; TG, triacylglycerol; MGDG, monogalactosyldiacylglycerol; CER, cerebrosides; DGDG, digalactosyldiacylglycerol; PG, phosphatidylglycerol; SQDG, sulphoquinovosyldiacylglycerol; PI, phosphatidylinositol; PE, phosphatidylethanolamine; PC, phosphatidylcholine.

In silver ion chromatography, the order of elution of triacylglycerol species is easily understood because only one property of the molecules is involved, i.e. degree of unsaturation. Reversed-phase chromatography (see below), on the other hand, effects separation both by chain-length and degree of unsaturation, each double bond reducing the effective chain-length by the equivalent of about two methylene groups. While good resolution is possible, chromatograms of unknown samples are not always easy to interpret.

When used in sequence the two techniques make a much more powerful tool. Fish oils gave highly complex chromatograms with reversed-phase HPLC, for example, and identification of individual components was impossible [29]. On the other hand, when fractions from silver ion HPLC were collected and then subjected to reversed-phase HPLC, separation was then in effect by chain-length only and the main peaks were easily identified. Each fraction was examined in turn in this way, and much more information was obtained in comparison to when either technique was used on its own. However, each fraction from the reversed-phase column still contained very many distinct molecular species, and HPLC in combination with mass spectrometry would be essential if individual components were to be identified let alone quantified.

Silver ion HPLC has considerable potential for the separation of positional and configurational isomers of fatty acids, and for quantitative determination of trans-unsaturation. To confirm that this was so, phenacyl derivatives of fatty acids were prepared so that accurate quantification with UV detection was possible [30]. Fortuitously, it now has become apparent that such derivatives give especially favourable separations. As an example, the separation obtained with phenacyl esters of the three main naturally occurring octadecenoic acid isomers (petroselinic, oleic and vaccenic acids) is illustrated in figure 3. Similarly, dichloroethanedichloromethane $(1: 1, \mathrm{v} / \mathrm{v})$ with a little acetonitrile added was used as the mobile phase to effect resolution of positional and configurational isomers of mono-, di- and trienoic 
fatty acids. The distance of the double bond from the carboxyl group was found to be more important in governing the separation of positional isomers than was the terminal region of the molecule [31]. As it was relatively easy to control the chromatographic parameters in silver ion HPLC in comparison with analogous TLC procedures, studies of the mechanism of the interaction between silver ions and unsaturated fatty acids now became possible. It was deduced that this form of silver ion HPLC involves simultaneous interactions between one silver ion and either two double bonds, or one double bond and one other electron-rich moiety such as the ester group [32].

\section{Chiral high-performance liquid chromatography}

One of the important stereochemical problem in the analysis of lipids is the resolution of enantiomeric 1,2- and 2,3diacyl-sn-glycerols, partly because of their importance as biosynthetic precursors of triacyl-sn-glycerols and partly as a step towards stereospecific analysis of the latter. Takagi and his colleagues in Japan used chiral phase HPLC columns and dinitrophenylurethane derivatives to obtain resolution of enantiomeric diacyl- and monoacylglycerols [33-36]. They are readily detected by their pronounced UV absorbance at $280 \mathrm{~nm}$ as they emerge from HPLC columns.

Another approach to the resolution of diacyl-sn-glycerols has been to convert them to diastereomeric urethane derivatives, such as the $(S)-(+)-1-(1-n a p h t h y l) e t h y l$ urethanes, by reaction with enantiomerically pure isocyanates. These diastereomers have now been resolved by HPLC on columns of silica gel [37]. This type of separation served as the basis for a novel procedure for stereospecific analysis of triacyl$s n$-glycerols from natural oils and fats, i.e. for determining the compositions of fatty acids esterified to each of positions $s n-1, s n-2$ and $s n-3[37,38]$. Triacyl-sn-glycerols were subjected to partial hydrolysis with a Grignard reagent and the products were converted to the $(S)-(+)-1-(1$-naphthyl)ethyl urethane derivatives. The diacylglycerol derivatives were isolated by a solid-phase extraction procedure and were resol-

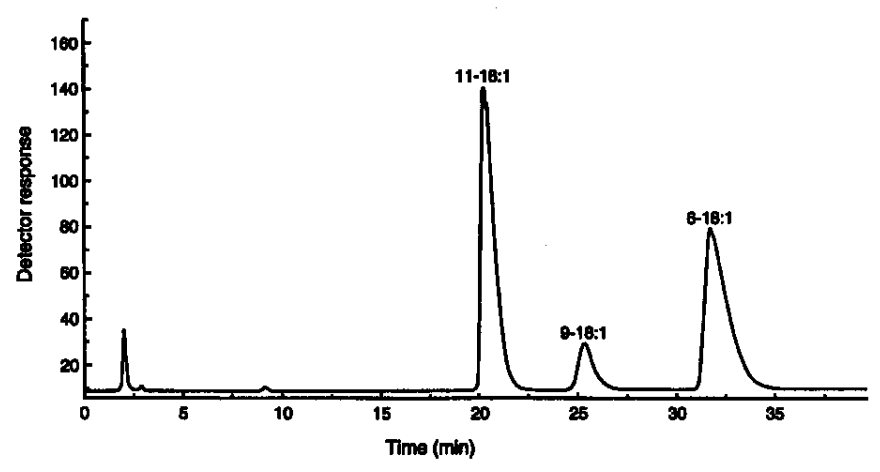

Figure 3. Separation of phenacyl esters of the isomeric octadecenoic acids, petroselinic (6-18:1), oleic (9-18:1) and vaccenic (11-18:1), by HPLC on a silver ion column (Nucleosil ${ }^{\mathrm{TM}}$ 5SA loaded with silver ions) eluted with dichloromethane-dichloroethane-acetonitrile (50:50:0.25 by volume) at a flow-rate of 1.5 $\mathrm{mL} / \mathrm{min}$, with evaporative light-scattering detection. ved into the diastereomeric forms by HPLC on silica gel. From high-precision GC analyses of the fatty acids from the intact triacylglycerols and of the 1,2- and 2,3-diacyl-sn-glycerol derivatives, the compositions of positions $s n-3$ and $s n$ 1 respectively could be calculated; that of position $s n-2$ was obtained by difference. Previously, this type of analysis could only be accomplished by time-consuming procedures involving a difficult synthetic step to prepare phosphatidylcholine, stereospecific hydrolyses with phospholipases and more complex separation procedures. The topic has been recently reviewed [39].

Chiral HPLC is proving particularly valuable as a means of resolving the hydroxy fatty acids produced by the action of lipoxygenases in the eicosanoid cascade [40].

\section{Reversed-phase high-performance liquid chromatography}

Procedures for separation of lipids into molecular species by reversed-phase HPLC are now well-established and robust and this is especially so of triacylglycerols (reviewed comprehensively elsewhere $[41,42]$ ). However, it is apparent that not all analysts are realizing the potential of the technique. A series of papers from the laboratory of Tchapla has demonstrated that columns of the octadecylsilyl type with a high carbon load, with acetonitrile-dichloromethane mixtures as mobile phase, are capable of giving the best results, especially when optimized by chemometrics methodology [44-46]. The nature of the separation is well known, i.e. that the retention time is dependent on the total number of carbon atoms in the three fatty acyl moieties, with each double bond reducing the effective chain-length of the species by the equivalent of about two carbon atoms. As mentioned briefly above, it therefore complements silver ion chromatography, where separation is based solely on degree of unsaturation, very well.

It has become apparent that atmospheric-pressure chemical ionization mass spectrometry in combination with reversed-phase HPLC is particularly well suited to analysis of triacylglycerols [47].

Reversed-phase HPLC is now also the most widely used method for analysing molecular species of phospholipids, as reviewed recently by Bell [48]. Methods are available for analysis of intact phospholipids or after removal of the polar head group. However, it is my opinion that the methodology would benefit from the chemometrics optimization approach used elsewhere for triacylglycerols.

\section{Supercritical-fluid chromatography}

Supercritical-fluid chromatography (SFC) lacks the resolving power of GC or HPLC, although the capacity to use flame-ionization detection gives it an advantage over the latter. In my opinion, the only exception is the use of SFC for separation of molecular species of triacylglycerols in routine screening applications. Here, the efficiency of the separation is comparable to that of high-temperature GC, but the separation is carried out at much lower temperatures and 
accurate quantification is possible. The technique can also be interfaced to atmospheric-pressure chemical ionization mass spectrometry. Some interesting examples have been published by Manninen and colleagues [49-51].

\section{Acknowledgement}

This paper is published as part of a programme funded by the Scottish Office Agriculture, Environment and Fisheries Dept.

\section{References}

1. Christie, W. W. Detectors for high-performance liquid chromatography of lipids with special reference to evaporative light-scattering detection. In: Advances in Lipid Methodology - One, Christie, W. W. Ed.; The Oily Press, Dundee, 1992; pp 239-271.

2. Laakso, P. Supercritical fluid chromatography of lipids. In: Advances in Lipid Methodology - One, Christie, W. W. Ed., The Oily Press, Dundee, 1992; pp 81-119.

3. Harvey, D. J. Mass spectrometry of picolinyl and other nitrogen-containing derivatives of fatty acids. In: Advances in Lipid Methodology - One, Christie, W. W. Ed., The Oily Press, Dundee, 1992; pp 19-80.

4. Christie, W. W. Structural analysis of fatty acids. In: Advances in Lipid Methodology - Four, Christie, W. W. Ed., The Oily Press, Dundee, 1997; pp 119-169.

5. Lie Ken Jie, M. S. F.; Choi, Y. C. Mass spectral determination of deuterium-labelled picolinyl fatty esters in the determination of double-bond position, J. Am. Oil Chem. Soc. 1992, 69, 1245-1247.

6. Lie Ken Jie, M. S. F.; Choi, Y. C. GC-MS of the picolinyl ester derivatives of deuterated acetylenic fatty acids, J. Chromatogr. 1992, 625, 271-276.

7. Francis, G. W. Alkylthiolation for the determination of double bond position in unsaturated fatty acid esters, Chem. Phys. Lipids 1991, 29, 369-374.

8. Vincenti, M.; Guglieilmetti, G.; Cassani, G.; Tonini, C. Determination of double bond position in diunsaturated compounds by mass spectrometry of dimethyl disulphide derivatives, Anal. Chem. 1987, 59, 694-699.

9. Mossoba, M. M.; McDonald, R. E.; Chen, J.-Y. T.; Armstrong, D. J.; Page, S. W. Identification and quantification of trans9,trans-12-octadecadienoic acid methyl ester and related compounds in hydrogenated soybean oil and margarines by capillary GC/matrix isolation/Fourier transform IR, J. Agric. Food Chem. 1990, 38, 86-92.

10. Mossoba, M. M.; McDonald, R. E.; Armstrong, D. J.; Page, S. W.; Hydrogenation of soybean oil: A TLC and GC/matrix isolation/Fourier transform infrared study, J. Agric. Food Chem. 1991, 39, 695-699.

11. Christie, W. W. Separation of phospholipid classes by highperformance liquid chromatography. In: Advances in Lipid Methodology - Three, Christie, W. W. Ed.; The Oily Press, Dundee, 1996; pp 77-107.

12. Patton, G. M.; Fasulo, J. M.; Robins, S. J. Separation of phospholipids and individual molecular species of phospholipids by HPLC, J. Lipid Res. 1982, 23, 190-196.

13. Rapid separation and quantification of lipid classes by HPLC and mass (light-scattering) detection, J. Lipid Res. 1985, 26, 507-512.

14. Christie, W. W. Separation of lipid classes by HPLC with the "mass detector", J. Chromato gr. 1986, 361, 396-399.
15. Mourey, T. H.; Oppenheimer, L. E. Principles of operation of an evaporative light-scattering detector for liquid chromatography, Anal. Chem. 1984, 56, 2427-2434.

16. Oppenheimer, L. E. Mour ey, T. H. Examination of the concentration response of evaporative light-scattering mass detector, J. Chromatogr. 1985, 323, 297-304.

17. Righezza, M.; Guiochon, G. Effects of the nature of the solvent and solutes on the response of a light-scattering detector, J. Liq. Chromatogr. 1988, 11, 1967-2004.

18. Christie, W. W.; Noble, R. C.; Davies, G. Phospholipids in milk and dairy products, J. Soc. Dairy Technol. 1987, 40, 1012.

19. Becart, J.; Chevalier, C.; Biesse, J. P. Quantitative analysis of phospholipids by HPLC with a light-scattering detector application to raw materials for cosmetic use, J. High Resolut. Chromatogr. 1990, 13, 126-129.

20. Breton, L.; Serkiz, B.; Volland, J. -P.; Lepagnol, J. A new rapid method for phospholipid separation by HPLC with light-scattering detection, J. Chromatogr. 1989, 497, 243-249.

21. Lutzke, B. S.; Braughler, J. M. An improved method for the identification and quantitation of biological lipids by HPLC using laser light-scattering detection, J. Lipid Res. 1990, 31, 2127-2130.

22. Conforti, F. D.; Harris, C. H.; Rinehart, J. T. HPLC analysis of wheat-flour lipids using an evaporative light-scattering detector, J. Chromatogr. 1993, 645, 83-88.

23. Moreau, R. A. Plant lipid class analysis by HPLC. In: Plant Lipid Biochemistry, Structure and Utilization, Quinn, P. J.; Harwood, J. L. Eds., Portland Press, London, 1990; pp 20-22.

24. Christie, W. W.; Urwin, R. A. Separation of lipid classes from plant tissues by HPLC on chemically bonded stationary phases J. High Resolut. Chromatogr. 1995, 18, 97-100.

25. Nikolova-Damyanova, B. Silver ion chromatography and lipids. In: Advances in Lipid Methodology - One, Christie, W. W. Ed., The Oily Press, Dundee, 1992; pp 181-237.

26. Christie, W. W. A stable silver-loaded column for the separation of lipids by HPLC, J. High Res. Chromatogr. Chromatogr. Commun. 1987, 10, 148-150.

27. Christie, W. W. Separation of molecular species of triacylglycerols by HPLC with a silver ion column, J. Chromatogr. 1988, 454, 273-284.

28. Laakso, P.; Christie, W. W.; Pettersen, J. W. Analysis of North Atlantic and Baltic fish oil triacylglycerols by HPLC with a silver ion column, Lipids 1990, 25, 284-291.

29. Laakso, P.; Christie, W. W. Combination of silver ion and reversed-phase HPLC in the fractionation of herring oil triacylglycerols, J. Am. Oil Chem. Soc. 1991, 68, 213-223.

30. Christie, W. W.; Breckenridge, G. H. M. Separation of cis and trans isomers of unsaturated fatty acids by HPLC in the silver ion mode, J. Chromatogr. 1989, 469, 261-269.

31. Nikolova-Damyanova, B.; Herslöf, B. G.; Christie, W. W. Silver ion high-performance liquid chromatography of derivatives of isomeric fatty acids, J. Chromato gr. 1992, 609, 133140.

32. Nikolova-Damyanova, B.; Christie, W. W.; Herslöf, B. Mechanistic aspects of fatty acid retention in silver ion chromatography, J. Chromatogr. A 1996, 749, 47-54

33. Takagi, T.; Ando, Y. Stereospecific analysis of triacyl-sn-glycerols by chiral HPLC, Lipids 1991, 26, 542-547.

34. Takagi, T. Chromatographic resolution of chiral lipid derivatives, Prog. Lipid Res. 1991, 29, 277-298.

35. Takagi, T. HPLC of enantiomeric acylglycerols and alkylglycerols. In: CRC Handbook of Chromatography. Analysis of Lipids, Mukherjee, K. D.; Weber, N. Eds., CRC Press, Boca Raton, 1993; pp 115-121.

36. Takagi, T.; Ando, Y. Stereospecific analysis of monounsaturated triacylglycerols in cocoa butter, J. Am. Oil Chem. Soc. 1995, 72, 1203-1206. 
37. Laakso, P.; Christie, W. W. Chromatographic resolution of chiral diacylglycerol derivatives: potential in the stereospecific analysis of triacyl-sn-glycerols, Lipids 1990, 25, 349-353.

38. Christie, W. W.; Nikolova-Damyanova, B.; Laakso, P.; Herslöf, B. Stereospecific analysis of triacyl-sn-glycerols via resolution of diastereomeric diacylglycerol derivatives by HPLC on silica, J. Am. Oil Chem. Soc. 1991, 68, 695-701.

39. Kuksis, A. Analysis of positional isomers of glycerolipids by non-enzymatic methods. In: Advances in Lipid Methodology Three, Christie, W. W. Ed., The Oily Press, Dundee, 1996; pp 1-36.

40. Christie, W. W. The chromatographic resolution of chiral lipids. In: Advances in Lipid Methodology - One, Christie, W. W. Ed., The Oily Press, Dundee, 1992; pp 121-148.

41. Heron, S.; Bleton, J.; Tchapla, A. Mechanism for separation of triacylglycerols in oils by liquid chromatography: identification by mass spectrometry, In: New Trends in Lipid and Lipoprotein Analyses, Sebedio, J.-L.; Perkins, E. G. Eds., AOCS Press, Champaign, 1995; pp 205-231.

42. Nikolova-Damyanova, B. Reversed-phase high-performance liquid chromatography: general principles and application to the analysis of fatty acids and triacylglycerols, In: Advances in Lipid Methodology - Four, Christie, W. W. Ed., The Oily Press, Dundee, 1997; pp 193-251.

43. Heron, S.; Tchapla, A. Role of the solvent in RPLC - influence of the nature of the modifier of the mobile phase in nonaqueous reverse-phase liquid chromatography, Analusis 1993, 21, 269-276.

44. Heron, S.; Tchapla, A. Using a molecular interaction model to optimize the separation of fatty triglycerides in reversed-phase liquid chromatography - fingerprinting the different types of fats, Oleag. Corps Gras Lipides 1994, 1, 219-228.

45. Heron, S.; Tchapla, A. Choice of stationary and mobile phases for separation of mixed triglycerides by liquid-phase chromatography, Analusis 1994, 22, 114-126.

46. Heinisch, S.; Lesellier, E.; Podevin, C.; Rocca, J. L.; Tchapla, A. Computerized optimization of RP-HPLC separation with nonaqueous or partially aqueous mobile phases, Chromatographia 1997, 44, 529-537.

47. Byrdwell, W. C.; Neff, W. E. Qualitative and quantitative analysis of triacylglycerols using atmospheric-pressure chemical ionization mass spectrometry, In: New Techniques and Applications in Lipid Analysis, McDonald, R. E.; Mossoba, M. M. Eds., AOCS Press, Champaign, 1997; pp 45-80.

48. Bell, M. Separations of molecular species of phospholipids by high-performance liquid chromatography, In: Advances in Lipid Methodology - Four, Christie, W. W. Ed., The Oily Press, Dundee, 1997; pp 45-82.

49. Manninen, P.; Laakso, P.; Kallio, H. Separation of g-linolenic and a-linolenic acid-containing triacylglycerols by capillary supercritical-fluid chromatography, Lipids 1995, 30, 665-671.

50. Manninen, P.; Laakso, P. Capillary supercritical fluid chromatography atmospheric pressure chemical ionization mass spectrometry of g-and a-linolenic acid containing triacylglycerols in berry oils, Lipids 1997, 32, 825-831.

51. Manninen, P.; Laakso, P. Capillary supercritical fluid chromatography atmospheric pressure chemical ionization mass spectrometry of triacylglycerols in berry oils, J. Am. Oil Chem. Soc. 1997, 74, 1089-1098. 